\title{
Nuclear receptor PXR: discovery of a pharmaceutical anti-target
}

\author{
Steven A. Kliewer \\ Departments of Molecular Biology and Pharmacology, University of Texas Southwestern Medical Center, Dallas, Texas, USA.

\begin{abstract}
Transcriptional induction of the gene encoding cytochrome P450 $3 \mathrm{~A}$ oxygenase (CYP3A) causes a prominent class of dangerous drug-drug interactions wherein one drug accelerates the metabolism of another. In our $1998 \mathrm{JCl}$ paper, we reported the cloning of the human nuclear receptor PXR and demonstrated that it mediates CYP3A induction. We determined that PXR is expressed in liver, acts through a DNA response element located in the CYP3A promoter, and is activated by a structurally diverse collection of drugs that induce CYP3A. Our findings revealed the molecular basis for the CYP3A induction class of drug-drug interactions and provided a highthroughput means for screening out drug candidates with this activity.
\end{abstract}

\section{CYP3A inducers and drug interactions}

In the 1960s, it was shown that repeated administration of "catatoxic" steroids to rodents conferred resistance against the harmful or anesthetic effects of structurally unrelated drugs (reviewed in ref. 1). For example, rats treated for several days with the anti-mineralocorticoid spironolactone were almost completely protected against otherwise fatal doses of the cardiotoxin digitoxin. Among the most potent of these catatoxic steroids was pregnenolone $16 \alpha$-carbonitrile (PCN). It was subsequently shown that PCN and other catatoxic steroids mediate their protective effects by inducing the transcription of hepatic oxygenases, including cytochrome P450 3A oxygenase (CYP3A), which metabolizes most prescription drugs. In an interesting twist, CYP3A induction was also shown to cause an important class of dangerous drug-drug interactions wherein the inducing drug accelerates the metabolism of other drugs that are CYP3A substrates (reviewed in refs. 2, 3).

At the time, the molecular basis for CYP3A induction was unknown; however, it was clear that the potential mechanism would have to account for two puzzling observations. First, there was a remarkable structural diversity among known chemical inducers of CYP3A, which included compounds as big as the macrocyclic antibiotic rifampicin (MW > 800) and, paradoxically, both nuclear receptor agonists and antagonists. Second, there were pronounced differences among species in CYP3A induction responses. For example, PCN robustly induces CYP3A in rodent but not human hepatocytes. Conversely, rifampicin induces CYP3A in human but not rodent hepatocytes. Studies with CYP3A promoter sequences and hepatocytes derived from different species showed that these speciesspecific differences were a consequence of host cell factors rather than differences in CYP3A promoters (reviewed in ref. 3). It appeared as though the mechanism underlying CYP3A induction was likely to be complex and involve multiple receptors.

\section{PXR: a promiscuous xenobiotic receptor}

The 1990s were an exciting time in the orphan nuclear receptor field, with ligands identified for retinoid $\mathrm{X}$ receptors (RXRs), PPARs, liver $\mathrm{X}$ receptors (LXRs), and farnesoid X receptor (FXR) (reviewed in refs. 4, 5). In 1997, our nuclear receptor drug discovery group at Glaxo Wellcome (now GlaxoSmithKline) came across an expressed sequence tag that encoded part of a novel nuclear receptor. We went on to clone this receptor and show that it was abundantly expressed in the liver. As part of our group's ongoing campaign to identify ligands for orphan nuclear receptors, we screened the receptor against a large library of compounds. Among our initial hits was PCN, which bound and activated the receptor at concentrations that were consistent with that required for CYP3A induction. We named this protein the pregnane $\mathrm{X}$ receptor (PXR) based on the efficient activation of the receptor by PCN and other natural and synthetic C21 steroids (pregnanes). We further demonstrated that PXR acted through a DNA response element located within the rodent Cyp3a gene promoter (6).

While we had identified the longsought-after PCN receptor, this work did not address the broader issue of the overall contribution of PXR to CYP3A induction and drug-drug interactions. This work did set the stage for our 1998 study published in the JCI, in which we cloned and characterized the human ortholog of PXR (7), which was subsequently reported by two other groups and referred to as steroid and xenobiotic receptor (SXR) (8) and PAR (9), respectively. The DNA-binding domain of mouse and human PXR shares $96 \%$ amino acid identity, and both orthologs bind the same response elements within CYP3A promoters. However, the ligand-binding domains are only $76 \%$ identical, which is much lower than the identity between orthologs of other nuclear receptors. This divergence provided the first hint that mouse and human PXR may have distinct pharmacologic activation profiles.

In cell-based reporter assays, human PXR was activated by a structurally diverse set of established CYP3A inducers, including the drugs rifampicin, dexamethasone, RU486, spironolactone, cyproterone acetate, clotrimazole, and lovastatin. Importantly, there were striking differences in 


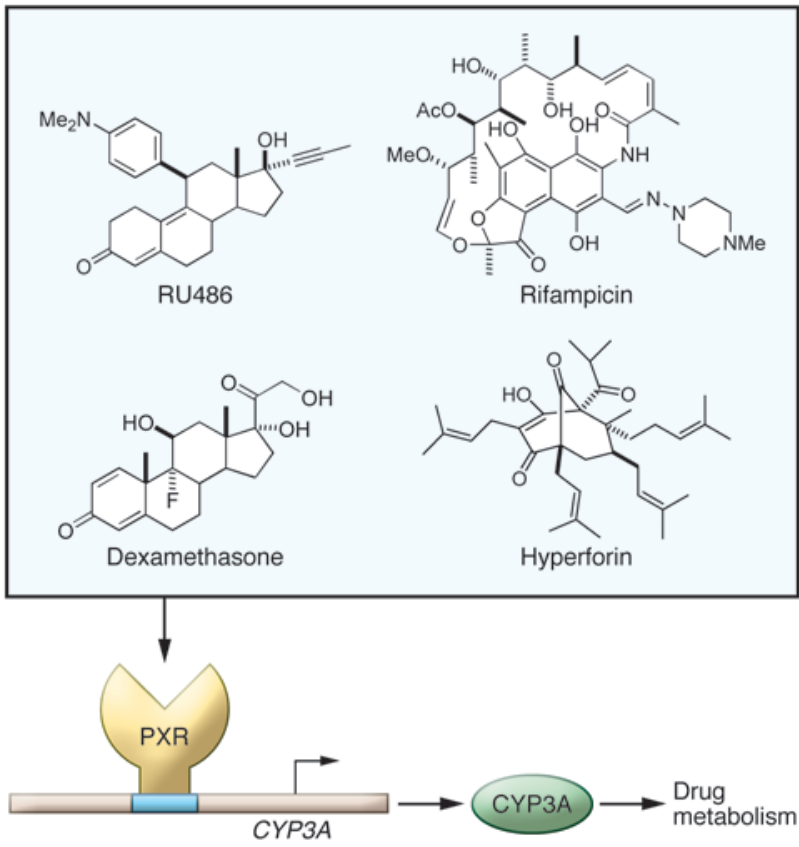

Figure 1. PXR causes drug-drug interactions. Due to a large, hydrophobic ligand-binding pocket, PXR is activated by a wide variety of drugs, including dexamethasone, RU486, rifampicin, and hyperforin (a constituent of St. John's wort). Once activated, PXR binds to the CYP3A promoter and induces transcription. Elevated levels of CYP3A result in increased metabolism of CYP3A substrates, which include most prescription drugs. the response profiles of mouse and human PXR that mirrored those for CYP3A induction in the two species. From these studies, we reached the surprising conclusion that most CYP3A induction was mediated by this single, divergent nuclear receptor (Figure 1). This conclusion was subsequently confirmed in an elegant study by the Evans laboratory that showed introduction of human PXR into mice results in a "humanized" CYP3A induction profile (10). Structural studies later revealed that PXR has a large, hydrophobic ligand-binding pocket that changes shape to accommodate different ligands (reviewed in ref. 11). Thus, PXR evolved to serve as a generalized xenobiotic sensor rather than a receptor for an endogenous ligand. This was a new concept in the nuclear receptor field.

The immediate impact of our JCI study was that high-throughput PXR assays, such as the one we used, could be applied prospectively to identify and eliminate CYP3A inducers early in the drug-discovery process. In fact, PXR assays are now widely used by pharmaceutical companies for this purpose. Notably, PXR is not only activated by prescription drugs, but also by herbs such as St. John's wort, which interacts with many drugs (reviewed in ref. 12).
Thus, PXR assays are also useful for assessing the potential of unregulated remedies to interact with prescription drugs.

In closing, our JCI paper identified PXR as a molecular basis for drug-drug interactions and introduced the concept of a nuclear receptor acting as a generalized xenobiotic sensor. Ironically, while our nuclear receptor group at Glaxo Wellcome set out to identify new drug targets, our pharmaceutical legacy is the discovery of an important anti-target!

\section{Acknowledgments}

This work was supported by the Robert A. Welch foundation (grant I-1558).

Address correspondence to: Steven A. Kliewer, Departments of Molecular Biology and Pharmacology, University of Texas Southwestern Medical Center, Room ND9.136G, 6001 Forest Park Road, Dallas, Texas 75390, USA. Phone: 214.645.6304; E-mail: steven.kliewer@ utsouthwestern.edu.

1. Kourounakis P, Selye H, Taché Y. Catatoxic steroids. Adv Steroid Biochem Pharmacol. 1977;6:35-57.

2. Gonzalez FJ, Liu SY, Yano M. Regulation of cytochrome P450 genes: molecular mechanisms.
Pharmacogenetics. 1993;3(1):51-57.

3. Quattrochi LC, Guzelian PS. Cyp3A regulation: from pharmacology to nuclear receptors. Drug Metab Dispos. 2001;29(5):615-622.

4. Kliewer SA, Lehmann JM, Willson TM. Orphan nuclear receptors: shifting endocrinology into reverse. Science. 1999;284(5415):757-760.

5. Chawla A, Repa JJ, Evans RM, Mangelsdorf DJ. Nuclear receptors and lipid physiology: opening the X-files. Science. 2001;294(5548):1866-1870.

6. Kliewer SA, et al. An orphan nuclear receptor activated by pregnanes defines a novel steroid signaling pathway. Cell. 1998;92(1):73-82.

7. Lehmann JM, McKee DD, Watson MA, Willson TM, Moore JT, Kliewer SA. The human orphan nuclear receptor PXR is activated by compounds that regulate CYP3A4 gene expression and cause drug interactions. JClin Invest. 1998;102(5):1016-1023.

8. Blumberg B, et al. SXR, a novel steroid and xenobiotic-sensing nuclear receptor. Genes Dev. 1998;12(20):3195-3205.

9. Bertilsson $\mathrm{G}$, et al. Identification of a human nuclear receptor defines a new signaling pathway for CYP3A induction. Proc Natl Acad Sci U S A. 1998;95(21):12208-12213.

10. Xie W, et al. Humanized xenobiotic response in mice expressing nuclear receptor SXR. Nature. 2000;406(6794):435-439.

11. Carnahan VE, Redinbo MR. Structure and function of the human nuclear xenobiotic receptor PXR. Curr Drug Metab. 2005;6(4):357-367.

12. Staudinger JL, Ding X, Lichti K. Pregnane X receptor and natural products: beyond drug-drug interactions. Expert Opin Drug Metab Toxicol. 2006;2(6):847-857. 